\title{
A UNIVERSAL FORMAL GROUP AND COMPLEX COBORDISM
}

\author{
BY MICHIEL HAZEWINKEL ${ }^{1}$
}

Communicated May 7, 1975

The purpose of this note is to 'announce' some of the results of [5], [6], [7] pertaining to formal groups and complex cobordism. These should have been written up a number of years ago. The phrase "formal group" is used as an abbreviation for commutative one-dimensional formal group (law).

1. Introduction. Below we give an explicit recursion formula for the logarithm of a universal commutative formal group and a $p$-typically universal commutative formal group. These give us a universal formal group $F_{U}$ defined over $\mathbf{Z}[U]=\mathbf{Z}\left[U_{2}, U_{3}, U_{4}, \ldots\right]$ and a $p$-typically universal formal group $F_{T}$ over $\mathrm{Z}\left[T_{1}, T_{2}, \ldots\right]$. Possibly the best way to look at these formal groups is as follows. To fix ideas let $p$ be a fixed prime number and let $A$ be a commutative ring with unit such that every prime number $\neq p$ is invertible in $A$. Let $F_{T}$ be the one-dimensional $p$-typically universal formal group and $G$ a one-dimensional formal group over $A$. Cartier [4] associates to $G$ a module of curves $C(G)$ over a certain ring $\operatorname{Cart}_{p}(A)$. The ring $\operatorname{Cart}_{p}(A)$ has as its elements expressions $\Sigma V^{i}\left[a_{i j}\right] \mathbf{f}^{j}, a_{i j} \in A$, which are added and multiplied according to certain rules, cf. [4] and [9]; $V$ stands for the 'Verschiebung' associated to the prime number $p$ and $\mathbf{f}$ stands for the 'Frobenius' associated to the prime number $p$. The left modules $C$ over $\operatorname{Cart}_{p}(A)$ which arise as modules of curves of some one-dimensional commutative formal group are of the form

$$
C \simeq \operatorname{Cart}_{p}(A) / \operatorname{Cart}_{p}(A)\left(\mathrm{f}-\sum_{i=1}^{\infty} V^{i}\left[t_{i}\right]\right), \quad t_{i} \in A .
$$

Now let $F_{t}$ be the formal group over $A$ obtained by substituting $t_{i}$ for $T_{i}$. Then $C\left(F_{t}\right)=C$.

2. The formulae. Choose a prime number $p$ and let

$$
l_{n}(T)=\sum T_{i_{1}} T_{i_{2}}^{p^{i_{1}}} \cdots T_{i_{s}}^{p^{i_{1}}+\cdots+i_{s-1}} / p^{s}
$$

where the sum is over all sequences $\left(i_{1}, i_{2}, \ldots, i_{s}\right), i_{j} \in \mathbf{N}=\{1,2,3, \ldots\}$ such that $i_{1}+\cdots+i_{s}=n$.

AMS (MOS) subject classifications (1970). Primary 14L05, 55 B20.

Key words and phrases. Universal formal group, complex cobordism, generators for $B P(p t)$ and $M U(p t)$.

1 Some of the results announced here were obtained in 1969/1970 while the author stayed at the Steklov Institute of Mathematics in Moscow and was supported by ZWO (the Netherlands Organization for advancement of Pure Research). 
Further, let

$$
\begin{gathered}
a_{n}(U)=\sum \frac{k\left(q_{1}, \ldots, q_{s}, d\right) \ldots k\left(q_{s}, d\right)}{p_{1} p_{2} \cdots p_{s}} \\
\cdot U_{q_{1}} U_{q_{2}}^{q_{1}} \cdots U_{q_{s}}^{q_{1} \cdots q_{s-1}} U_{d}^{q_{1} \cdots q_{s}}
\end{gathered}
$$

where we take $U_{d}=1$ if $d=1$; the sum is over all sequences $\left(q_{1}, \ldots, q_{s}, d\right)$ with $q_{i}=p_{i}^{r_{i}}, p_{i}$ a prime number, $r_{i} \in \mathbf{N}$ and $d=1$ or $d>1$ and not a power of a prime number; the integers $k\left(q_{1}, \ldots, q_{s}, d\right)$ can be chosen arbitrarily subject to the following congruences:

$$
\begin{gathered}
k\left(q_{1}, \ldots, q_{s}, d\right) \equiv\left\{\begin{array}{l}
1 \bmod p_{1} \\
0 \bmod p_{2}^{j-1}
\end{array} \text { if } q_{1}=p_{1}^{r_{1}}, q_{2}=p_{2}^{r_{2}}, \ldots, q_{j}=p_{2}^{r_{j}},\right. \\
p_{1} \neq p_{2}, q_{j+1} \text { not a power of } p_{2}
\end{gathered}
$$

a power of $p_{1}$.

We now define

$$
f_{T}(X)=\sum_{n \geqslant 0} l_{n}(T) X^{p^{n}}, \quad f_{U}(X)=\sum_{n \geqslant 1} a_{n}(U) X^{n},
$$

where we take $l_{0}(T)=1$ and $a_{1}(U)=1$.

One has the following recursion formula for the $T_{i}$ in terms of the $l_{i}$

$$
p l_{n}(T)=l_{n-1}(T) T_{1}^{p^{n-1}}+l_{n-2}(T) T_{2}^{p^{n-2}}+\cdots+l_{1}(T) T_{n-1}^{p}+T_{n}
$$

The situation for the $a_{i}$ and $U_{i}$ is slightly more complicated. We have

$$
\nu(n) a_{n}(U)=U_{n}+\sum_{i=1}^{\infty}(-1)^{i+1} \sum^{(i)} \rho\left(n, d_{1}\right) a_{d}(U) U_{d_{i}}^{d} U_{d_{i-1}}^{d d_{i}} \cdots U_{d_{1}}^{d d_{i}} \cdots d_{2}
$$

if we choose the $k\left(q_{1}, \ldots, q_{s}, d\right)$ in a certain special way (cf. [5, part II]). Here $\Sigma^{(i)}$ is the sum over all sequences $\left(d, d_{i}, d_{i-1}, \ldots, d_{1}\right)$ such that $d, d_{i}$, $\ldots, d_{1} \in \mathbf{N}, d_{1} \neq 1, s, d_{j}>1$ and not a power of a prime number for $j=2$, $\ldots, i$ and $d d_{i} \cdots d_{1}=s$. (Note that there are contributions with $d=1$ in $\Sigma^{(i)}$ if $i \geqslant 2$ but no contributions with $d=1$ in $\Sigma^{(1)}$.) The numbers $v(n)$ and $\rho\left(n, d_{1}\right)$ which occur in (2.6) are obtained as follows. For every pair of prime numbers let $c\left(p, p^{\prime}\right)$ be an integer such that $c(p, p)=1, c\left(p, p^{\prime}\right) \equiv 1 \bmod p$ and $c\left(p, p^{\prime}\right) \equiv 0 \bmod p^{\prime}$ if $p \neq p^{\prime}$. Now for all $(s, d)$ such that $d / s$ we define: $r(s, d)=1$ if $d=1$ or $d>1$ and not a power of a prime number, $r\left(s, p^{r}\right)=$ $\Pi c\left(p^{\prime}, p\right)$ where the product is over the set prime numbers $p^{\prime}$ which divide $s$. We define $\nu(n)=1$ if $n=1$ or $n>1$ and not a power of a prime number and $\nu\left(p^{r}\right)=p$ if $r \in \mathbf{N} . \rho(s, d)$ is now defined as $\nu(s) \nu(d)^{-1} r(s, d)$. 
3. Universality theorems. We define

$$
F_{U}(X, Y)=f_{U}^{-1}\left(f_{U}(X)+f_{U}(Y)\right), \quad F_{T}(X, Y)=f_{T}^{-1}\left(f_{T}(X)+f_{T}(Y)\right)
$$

where $f_{U}^{-1}$ and $f_{T}^{-1}$ are the inverse power series to $f_{U}$ and $f_{T}$; i.e. $f_{U}^{-1}\left(f_{U}(X)\right)=$ $X$ and similarly for $f_{T}$. One now has

3.2. TheOREM. $F_{T}(X, Y)$ is a formal power series with coefficients in $\mathrm{Z}\left[T_{1}, T_{2}, \ldots\right] . F_{U}(X, Y)$ is a formal power series with coefficients in $\mathbf{Z}\left[U_{2}, U_{3}, \ldots\right]$.

The two power series hence define commutative formal groups over $\mathbf{Z}[T]$ and $\mathbf{Z}[U]$.

3.3. THEOREM. $F_{U}$ is a universal formal group. $F_{T}$ is a p-typically universal formal group.

I.e. if $G(X, Y)$ is any formal group (resp. $p$-typical formal group) over a commutative ring with unit $A$, then there is a unique homomorphism $\phi: Z[U]$ $\rightarrow A$ (resp. $\phi: \mathbf{Z}[T] \rightarrow A$ ) such that $G(X, Y)$ is equal to the formal group obtained from $F_{U}$ (resp. $F_{T}$ ) by applying $\phi$ to its coefficients.

There are more dimensional analogues for the $F_{U}$ and $F_{T}$ and corresponding more dimensional analogues of Theorems 3.2 and 3.3. Cf. [5].

\section{Application to complex cobordism and Brown-Peterson cohomology.} Let $M U$ denote the unitary (co)bordism spectrum and $B P$ the Brown-Peterson spectrum. The associated cohomology theories are complex oriented and hence define groups over $M U(p t)$ and $B P(p t)$. The logarithms of these formal groups are by [11], [12], cf. also [1, part II], equal to

$$
\begin{aligned}
\log \mu_{M U}(X) & =\sum_{n \geqslant 0} m_{n} X^{n+1}, \\
\log \mu_{B P}(X) & =\sum_{n \geqslant 0} m_{p^{n-1}} X^{p^{n}}
\end{aligned}
$$

with $m_{n}=(n+1)^{-1}\left[\mathbf{C P}^{n}\right]$, where $\mathbf{C P}^{n}$ is the complex projective space of (complex) dimension $n$, and $m_{0}=1$. By [12], cf. also [1], we have that the formal group $\mu_{M U}$ is universal and that $\mu_{B P}$ is $p$-typically universal.

Hence there are uniquely determined isomorphisms $\phi: \mathbf{Z}[U] \rightarrow M U(p t)$ and $\Psi: \mathbf{Z}[T] \rightarrow B P(p t)$ taking (2.2) and (2.1) into (4.1). It follows that the $\phi\left(U_{2}\right), \phi\left(U_{3}\right), \ldots$ are a free polynomial basis for $M U(p t)$ and that the $\Psi\left(T_{1}\right)$, $\Psi\left(T_{2}\right), \ldots$ are a free polynomial basis for $B P(p t)$. Knowing $\log \mu_{M U}$ and $\log \mu_{B P}$ we can calculate these $\varphi\left(U_{n}\right)$ and $\Psi\left(T_{n}\right)$ by means of formulae (2.6) and (2.5). We find $B P(p t) \simeq \mathbf{Z}_{(p)}\left[v_{1}, v_{2}, \ldots\right], M U(p t)=\mathbf{Z}\left[u_{2}, u_{3}, \ldots\right]$ with the $v_{i}$ and $u_{i}$ related to the $m_{i}$ by the formulae:

$$
p m_{p^{n-1}}=m_{p^{n-1-1}} v_{1}^{p^{n-1}}+m_{p^{n-2}-1} v_{2}^{p^{n-2}}+\cdots+m_{p-1} v_{n-1}^{p}+v_{n},
$$


(4.3) $\nu(n) m_{n-1}=u_{n}+\sum_{i=1}^{\infty}(-1)^{i} \sum^{(i)} \rho\left(n, d_{1}\right) m_{d-1} u_{d_{i}}^{d} u_{d_{i-1}}^{d d_{i}} \cdots u_{d_{1}}^{d d_{i} \cdots d_{2}}$.

$B P$ is a direct summand of $M U \mathbf{Z}_{(p)}$, where $\mathrm{Z}_{(p)}$ denotes the integers localized at $p$. Because formula (4.3) reduces to (4.2) if $n=p^{s}$ under the identification $v_{i}=$ $u_{p} i$, we see that the $v_{i}$ are integral i.e. they live in $M U(p t)$ not just in $M U Z_{(p)}(p t)$. Cf. also [2].

Acknowledgement. Liulevicius [10] was the first to write down a formula similar to (4.2) and to prove that it gives generators for $B P(p t)$ in the case $p=2$.

\section{REFERENCES}

1. J. F. Adams, Stable homotopy and generalized homology, Univ. of Chicago Press, Chicago, Ill., 1974.

2. J. C. Alexander, On Liulevicius' and Hazewinkel's generators for $\pi_{*}(B P)$, Univ. of Maryland (preprint).

3. S. Araki, Typical formal groups in complex cobordism and $K$-theory, Lectures in Math., 6, Kyoto University, 1973.

4. P. Cartier, Modules associés à un groupe formel commutatif. Courbes typiques, C. R. Acad. Sci Paris Sér. A-B 265 (1967), A129-A132. MR 36 \#1449.

5. M. Hazewinkel, Constructing formal groups. I, II, III, IV, Reports 7119, 7201, 7207, 7322, Econometric Institute, Erasmus Univ., Rotterdam.

6. - Some of the generators of the complex cobordism ring, Report 7412, Econometric Institute, Erasmus Univ., Rotterdam.

7. - On operations in Brown-Peterson cohomology, Report 7502, Econometric Institute, Erasmus Univ., Rotterdam.

8. J. Kozma, Witt vectors and complex cobordism, Topology 13 (1974), 389-394.

9. M. Lazard, Sur les théorèmes fondamentaux des groupes formels commutatifs. I, II, Nederl. Akad. Wetensch. Proc. Ser. A 76 = Indag. Math. 35 (1973), 281-290, 291-300. MR 48 \#11129a,b,

10. A. L. Liulevicius, On the algebra $B P_{*}(B P)$, Lecture Notes in Math., vol. 249, Springer-Verlag, New York, 1972, pp. 47-52.

11. S. P. Novikov, The method of algebraic topology from the viewpoint of cobordism theories, Izv. Akad. Nauk Ser. Mat. 31 (1967), 855-951 = Math. USSR Izv. 1 (1967), 827-922. MR 36 \#4561.

12. D. Quillen, On the formal group laws of unoriented and complex cobordism theory, Bull. Amer. Math. Soc. 75 (1969), 1293-1298. MR 40 \#6565.

DEPARTMENT OF MATHEMATICS, ECONOMETRIC INSTITUTE, ERASMUS UNIVERSITY ROTTERDAM, ROTTERDAM, THE NETHERLANDS 